\title{
Severe Gingival Swelling and Erythema
}

Mohammed Bindakhil, DDS; Thomas P. Sollecito, DMD; Eric T. Stoopler, DMD

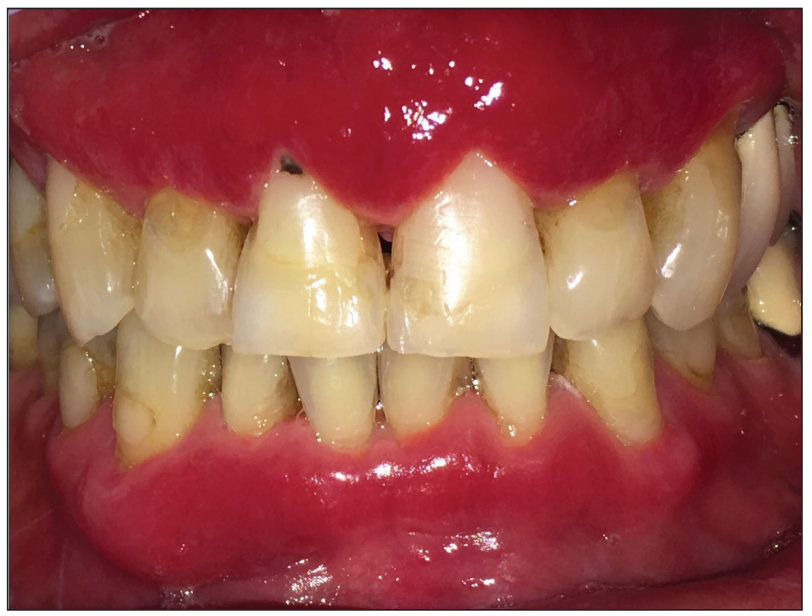

A 62-year-old man presented to an oral medicine specialist with gingival inflammation of at least 1 year's duration. He reported mild discomfort when consuming spicy foods and denied associated extraoral lesions. His medical history revealed hypertension, hypothyroidism, and psoriasis. Medications included lisinopril $10 \mathrm{mg}$ and levothyroxine $100 \mu \mathrm{g}$ daily. No known drug allergies were reported. His family and social history were noncontributory, and a detailed review of systems was unremarkable. Extraoral examination revealed no lymphadenopathy, salivary gland enlargement, or thyromegaly. Intraoral examination revealed diffuse enlargement of the maxillary and mandibular gingiva accompanied by severe erythema and bleeding on provocation. A 3-mm punch biopsy of the gingiva was performed for routine analysis and direct immunofluorescence.

\section{WHAT'S YOUR DIAGNOSIS?}
a. extramedullary plasmacytoma
b. mucous membrane pemphigoid
c. oral lichen planus
d. pemphigus vulgaris
e. plasma cell gingivitis

From the Department of Oral Medicine, University of Pennsylvania School of Dental Medicine, Philadelphia. 


\section{THE DIAGNOSIS: Plasma Cell Gingivitis}

M icroscopic analysis demonstrated an acanthotic stratified squamous epithelium with an edematous fibrous stroma containing dense perivascular infiltrates of plasma cells and lymphocytes (Figure 1). Immunohistochemical analysis with kappa, lambda, and CD79a immunostains indicated a polyclonal proliferation of plasma cells that excluded monoclonal plasma cell neoplasia (Figure 2). Direct immunofluorescence (DIF) was negative. Serum enzyme-linked immunosorbent assay for bullous pemphigoid 180 and 230 antibodies as well as desmoglein 1 and 3 antibodies was normal. The cumulative findings were consistent with plasma cell gingivitis (PCG). It was recommended that the patient avoid possible foods (eg, citrus) and oral hygiene products (eg, mint-flavored toothpaste) that could trigger PCG. With patient compliance to an elimination diet for 3 months, the condition resolved (Figure 3).

Plasma cell gingivitis is a rare condition characterized by generalized edema and erythema of the attached gingiva. It was described in the 1960s and classified into 3 types based on etiology: (1) hypersensitivity (most common), (2) neoplastic, and (3) PCG of unknown origin. ${ }^{1,2}$ Spices, herbs, and flavoring agents are implicated as potential triggers of hypersensitivity PCG, while neoplastic PCG is associated with monoclonal plasma cell neoplasms, such as multiple myeloma and extramedullary plasmacytoma. ${ }^{2,3}$ Histologically, a diffuse subepithelial infiltrate of a polyclonal mixture of plasma cells typically is observed in hypersensitivity PCG. ${ }^{3}$ The plasma cell infiltration in hypersensitivity PCG is a benign reactive process without known risk for development of plasma cell malignancy, but the presence of a notable number of plasma cells may require special tissue staining to rule out the possibility of associated neoplasia. ${ }^{2,3}$ There are no standardized protocols for management of PCG. ${ }^{4}$ Elimination of potential allergens, including flavored oral hygiene products, may result in resolution of hypersensitivity PCG lesions, as exemplified in our patient. ${ }^{1}$ Neoplastic PCG responds to treatment of the underlying malignancy. ${ }^{5}$ Topical, intralesional, and/ or systemic steroids may be considered in symptomatic cases of PCG. ${ }^{4}$

Clinical presentation of PCG can mimic immunemediated mucocutaneous diseases such as mucous membrane pemphigoid (MMP), pemphigus vulgaris (PV), and oral lichen planus; microscopic analysis is needed to establish the diagnosis. ${ }^{6}$ Mucous membrane pemphigoid is a chronic autoimmune blistering disease involving the mucous membranes with possible cutaneous involvement. It is characterized by a complement-mediated autoantibody process against one or several antigens in

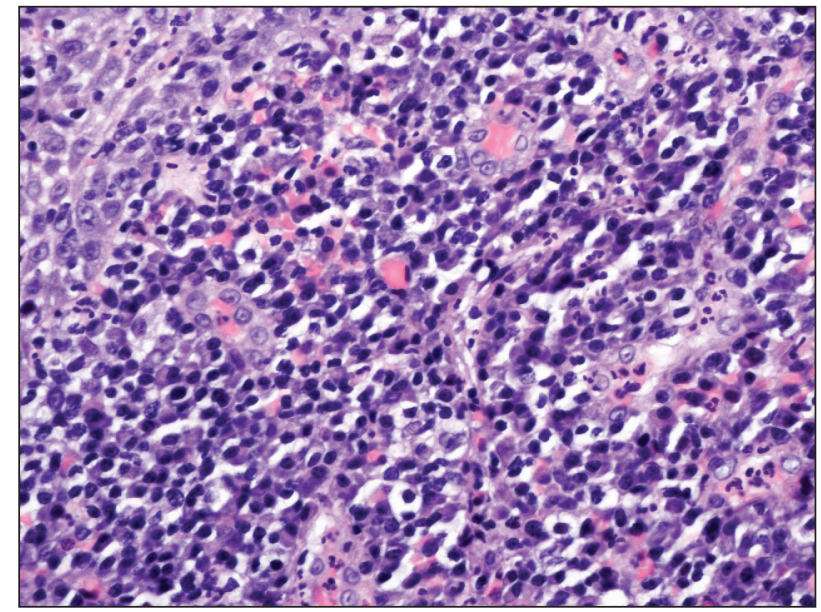

FIGURE 1. Plasma cell gingivitis. Dense inflammatory infiltrate consisting predominantly of plasma cells with scattered lymphocytes and neutrophils $(H \& E$, original magnification $\times 40)$.

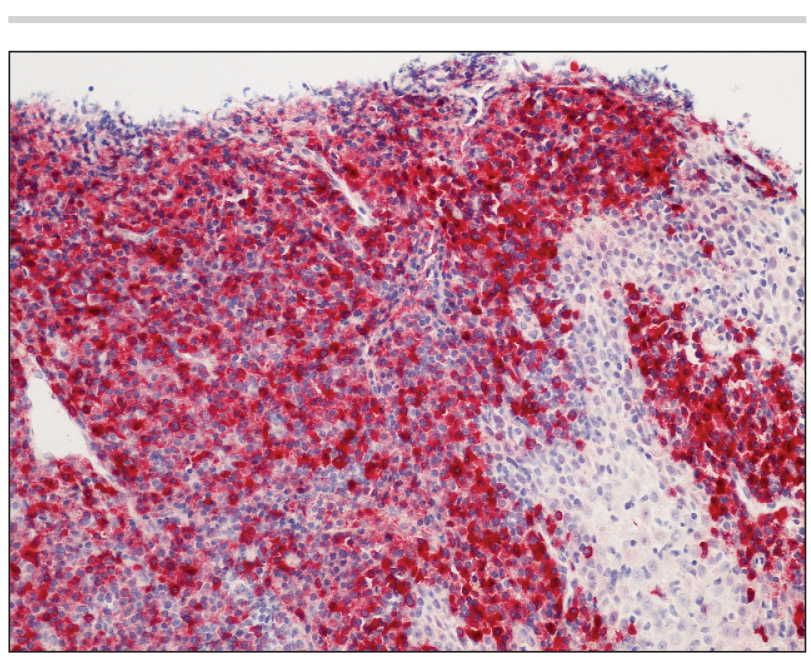

FIGURE 2. Plasma cell gingivitis. Abundant plasma cells stained positive for CD79a (original magnification $\times 25$ ).

the epithelial basement membrane. The oral mucosa is involved in $85 \%$ of MMP patients, and $65 \%$ of patients experience complications involving the ocular conjunctiva. Intraorally, MMP typically manifests as painful erosions, ulcerations, desquamative gingivitis, and/or occasionally intact blisters. Ocular complications include conjunctivitis and corneal erosions that often scar, resulting in blindness in approximately $15 \%$ of patients with ocular involvement. Microscopic features of MMP classically exhibit subepithelial separation with a mixed inflammatory cell infiltrate on routine analysis and linear deposition of IgG, IgA, or C3 within the basement membrane zone on DIF. 

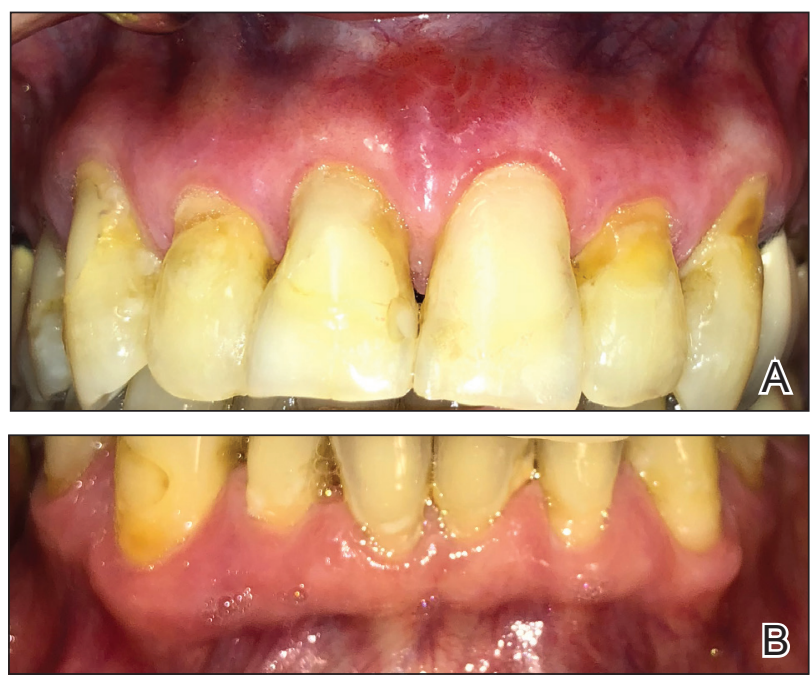

FIGURE 3. A and B, Maxillary and mandibular gingiva after a 3-month elimination diet of potential triggers for plasma cell gingivitis.

Treatment of MMP involves topical or systemic immunosuppressants to control symptoms, minimize complications, and alter disease progression. ${ }^{6}$

Pemphigus vulgaris is an autoimmune vesiculobullous disease that affects the oral mucosa with or without cutaneous involvement. ${ }^{7}$ Desmogleins 1 and 3, transmembrane glycoproteins of desmosomes that convene cell-to-cell adhesion, are identified as antigens in PV. Antibodies against these desmoglein proteins result in intraepithelial separation, which leads to blister formation. ${ }^{7}$ Oral manifestations of PV include mucosal erosions and ulcerations as well as desquamative gingivitis. Bullae rarely are seen in the oral cavity, as they tend to rupture, leaving nonhealing ulcerations. ${ }^{8}$ Histologically, PV is characterized by acantholysis of the suprabasal cell layers with an intact basement membrane zone on routine examination. The distinctive microscopic feature of $\mathrm{PV}$ is the detection of cell surface-bound IgG within the epidermis on DIF. Treatment of PV may include topical and/or systemic corticosteroids and other immunosuppressants. Rituximab, a monoclonal antibody, has been successful in the management of $\mathrm{PV} .^{8}$

Oral lichen planus is a T-cell mediated autoimmune condition that leads to subepithelial lymphocytic infiltration and excessive keratinocyte apoptosis. ${ }^{9}$ Women typically are affected more often than men, and $75 \%$ of patients also have cutaneous manifestations of the condition. Desquamation and/or erythema of the gingiva may be the initial manifestation of oral lichen planus. ${ }^{9}$ Other commonly involved sites include the buccal mucosa, tongue, and palate. Biopsy of affected tissues typically demonstrates degeneration of the basal cell layer with subjacent bandlike lymphocytic infiltration on routine staining. Linear fibrinogen at the basement membrane zone usually is observed on DIF. Topical corticosteroids are considered first-line therapy, but systemic therapy including corticosteroids, steroid-sparing agents, or immunomodulators may be used in severe cases. ${ }^{9}$

There are 3 variants of plasma cell neoplasms including multiple myeloma, medullary plasmacytoma (also known as solitary bone plasmacytoma), and extramedullary plasmacytoma (EMP). ${ }^{10}$ Extramedullary plasmacytoma, sometimes referred to as extraosseous plasmacytoma, is described as a solitary or multiple plasma cell neoplasm contained in the soft tissue. Its occurrence is rare, accounting for only $3 \%$ of plasma cell neoplasms. Approximately $90 \%$ of EMPs affect the head and neck region, and males are affected 4 times more often than females. The oral cavity is one of the sites of clinical presentation; the gingival tissue infrequently is affected. When EMP affects the gingiva, it can mimic any form of gingivitis as well as other benign inflammatory conditions, such as pyogenic granuloma. Biopsy is the gold standard diagnostic method for differentiating EMP from other conditions, and specific immunohistochemical stains are essential for the diagnosis. Extramedullary plasmacytoma has the best prognosis among plasma cell neoplasms, despite the risk for progression to multiple myeloma. Extramedullary plasmacytoma lesions are very sensitive to radiotherapy, and the 10 -year survival rate is approximately $70 \% .{ }^{10}$

\section{REFERENCES}

1. Sollecito TP, Greenberg MS. Plasma cell gingivitis: report of two cases. Oral Surg Oral Med Oral Pathol. 1992;73:690-693.

2. Gargiulo AV, Ladone JA, Ladone PA, et al. Case report: plasma cell gingivitis A. CDS Rev. 1995;88:22-23.

3. Abhishek K, Rashmi J. Plasma cell gingivitis associated with inflammatory cheilitis: a report on a rare case. Ethiop J Health Sci. 2013;23:183-187.

4. Arduino PG, D'Aiuto F, Cavallito C, et al. Professional oral hygiene as a therapeutic option for pediatric patients with plasma cell gingivitis: preliminary results of a prospective case series. J Periodontol. 2011;82:1670-1675

5. Nayak A, Nayak MT. Multiple myeloma with an unusual oral presentation. J Exp Ther Oncol. 2016;11:199-206.

6. $\mathrm{Xu} \mathrm{HH}$, Werth VP, Parisi E, et al. Mucous membrane pemphigoid. Dent Clin North Am. 2013;57:611-630.

7. Hammers CM, Stanley JR. Mechanisms of disease: pemphigus and bullous pemphigoid. Ann Rev Pathol. 2016;11:75-97.

8. Cizenski JD, Michel P, Watson IT, et al. Spectrum of orocutaneous disease associations: immune-mediated conditions. J Am Acad Dermatol. 2017;77:795-806.

9. Stoopler ET, Sollecito TP. Recurrent gingival and oral mucosal lesions. JAMA. 2014;312:1794-1795

10. Nair SK, Faizuddin M, Jayanthi D, et al. Extramedullary plasmacytoma of gingiva and soft tissue in neck. J Clin Diagn Res. 2014;8:ZD16-ZD18. 